\title{
Compact optical microfiber phase modulator
}

\author{
Xueliang Zhang $^{1,2, *}$, M. Belal ${ }^{1}$, G. Y. Chen ${ }^{1}$, Zhangqi Song ${ }^{2}$, G. Brambilla ${ }^{1}$, and T. P. Newson ${ }^{1}$ \\ ${ }^{1}$ Optoelectronics Research Centre, University of Southampton, Southampton, SO17 1BJ, UK \\ ${ }^{2}$ College of Optoelectronic Science and Engineering, National University of Defense Technology, Changsha 410073, China \\ ${ }^{*}$ Corresponding author: xz2d10@orc.soton.ac.uk
}

Received October 28, 2011; revised November 25, 2011; accepted November 28, 2011; posted November 29, 2011 (Doc. ID 157354); published January 19, 2012

\begin{abstract}
A compact optical microfiber phase modulator with $\mathrm{MHz}$ bandwidth is presented. A micrometer-diameter microfiber is wound on a millimeter-diameter piezoelectric ceramic rod with two electrodes. When a voltage is applied to the piezoelectric ceramic, the rod is strained, leading to a phase change along the microfiber; because of the small size, the optical microfiber phase modulator can have as high as a few MHz bandwidth response. @ 2012 Optical Society of America

OCIS codes: $\quad 060.2430,120.5060,250.4110$.
\end{abstract}

Fiber optic phase modulators are important components in the area of fiber communications and sensing. In general, they can be classified into two categories: (1) components (mainly including electro-optic [1] and thermo-optic [2] phase modulators) in which the fiber is only used as transmission line and (2) devices which are based on the optical phase change along the fiber and can exploit the stretching of functional materials such as piezoelectric transducer (PZT) [3,4] or magnetostrictive compounds [5]. The latter is simpler and has lower cost, but also tends to have lower frequency response than the former. Although fiber modulators with PZT film coating reported in the literature [ $\underline{6}-\underline{8}]$ have frequency responses higher than $1 \mathrm{MHz}$, they are relatively expensive and typically very long, in some cases in excess of $100 \mathrm{~mm}$.

Optical microfibers (OMs) have recently become increasingly attractive because of their enabling optical properties [9], which include convenient connectivity to other fiberized components, strong optical confinement, and good flexibility. Moreover, OMs have such low bending loss that they can be wound on narrow PZT rods modulated at high frequencies. In this Letter, an optical phase modulator based on a microfiber wound on a $1 \mathrm{~mm}$ diameter PZT is proposed and demonstrated. This device can reach frequency responses in excess of $1 \mathrm{MHz}$, is potentially low-cost and has a compact size smaller than $2 \mathrm{~mm}$ in any direction.

It is known that the working frequency of bulk PZTs is related to its geometry and to the material frequency constants. The high bending loss of conventional fibers (including bend insensitive fibers) restricts the bend radius to $\sim 5 \mathrm{~mm}$, thus limiting the total size of a PZT coiled fiber optical phase modulator. On the other hand, the excellent OM bending properties allow for a considerable reduction in size, permitting the construction of high bandwidth phase modulators. Although the mechanical properties of $\mathrm{OM}$ allow for extremely small bend radii [10], light transmission provides the main restriction to bend radius, especially when OMs are embedded in low refractive index polymers. According to [11] , the relationship between the bending loss for unit length 1 meter $(\alpha)$ and the bend radius $(R)$ for a single-mode OM embedded in low refractive index polymer can be expressed as $\alpha=-10 \log \left\{1-\frac{\sqrt{\pi} k_{0}^{2}\left(n_{1}^{2}-n_{\mathrm{eff}}^{2}\right) \exp \left[-\frac{2}{3} k_{0} \frac{\left(n_{\mathrm{eff}}^{2}-n_{2}^{2}\right)^{3 / 2}}{n_{\mathrm{eff}}^{2}} R\right]}{2 \gamma^{3 / 2} V^{2} \sqrt{R} K_{0}(\gamma a) K_{2}(\gamma a)}\right\}$,

where $k_{0}=2 \pi / \lambda$ is the free space propagation constant, $\lambda$ is the light wavelength in vacuum, $n_{1}$ and $n_{2}$ are the microfiber and the coating refractive indices, $n_{\text {eff }}$ is the effective index of the mode propagating in the OM, $a$ is the radius of the microfiber, $V$ is normalized frequency of the microfiber embedded in the polymer:

$$
V=k_{0} a\left(n_{1}^{2}-n_{2}^{2}\right)^{1 / 2} ; \gamma=k_{0}\left(n_{\mathrm{eff}}^{2}-n_{2}^{2}\right)^{1 / 2}
$$

and $K_{0}$ and $K_{2}$ are the zero- and second-rank modified Hankel functions. Here, the coating thickness is assumed to be infinite compared with the size of the microfiber diameter and other losses such as the scattering and absorption are neglected. Equation (1) is valid only provided that the bend radius does not lead to distorted field [11] along the OM.

Figure 1 shows the relationship between the radius of the PZT cylinder and the OM bending loss $\alpha$ (unit: $\mathrm{dB} / \mathrm{mm}$ ) at the wavelength of $\lambda=1.55 \mu \mathrm{m}$ obtained from Eq. (1), for a silica OM with $2 \mu$ m diameter coated with a polymer having $n_{2}=1.37$. It is evident that the OM has an acceptable level of loss even when $R=0.5 \mathrm{~mm}$ (bending

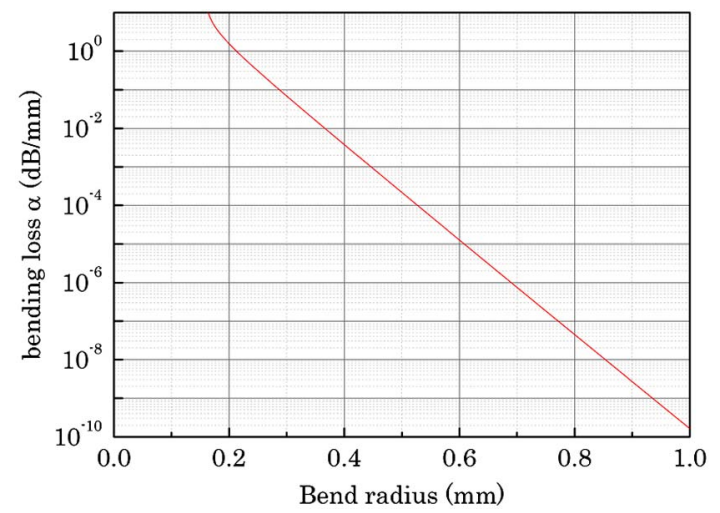

Fig. 1. (Color online) Bending loss of a $2 \mu \mathrm{m}$ diameter silica optical microfiber embedded into a polymer with refractive in$\operatorname{dex} n_{2}=1.37$ at wavelength $\lambda=1550 \mathrm{~nm}$. 
loss is smaller than $1 \mathrm{~dB}$ for 1 meter OM wound around a cylinder with $0.5 \mathrm{~mm}$ radius). In comparison, a conventional optical fiber with $125 \mu \mathrm{m}$ diameter cannot cope with such small bend radii due to not only the optical but also the mechanical properties of the fiber. When $R>0.5 \mathrm{~mm}$, the loss of wound OM microfiber embedded in polymer may be ignored. Pronounced surface roughness can also yield large bend losses, thus particular care has to be taken to coat the small diameter PZT cylinder with a uniform layer of low refractive index coating.

An OM has two pigtails, thus two conical transition regions where the diameter is gradually increasing. If the conical part is wound around the PZT cylinder, it will produce high loss [11]. Hence, only the uniform part of the microfiber can be wound around the cylinder. Figure 1 shows that millimeter-size PZT microfiber optic phase modulator may be realized.

The experimental demonstration of an OM PZT modulator was carried out, using a $2 \mu \mathrm{m}$ diameter $\mathrm{OM}$ with about 25-mm-long uniform waist region drawn from a conventional single-mode fiber, which was wound around a Teflon coated PZT rod of overall diameter $1.5 \mathrm{~mm}$. A PZT rectangular prism with dimensions $1 \mathrm{~mm} \times 1 \mathrm{~mm} \times 6 \mathrm{~mm}$ was cut from a bulk PZT plate with $1 \mathrm{~mm}$-thickness, which had two silver electrodes on the top and bottom sides. The electrodes were connected to copper wire for electrical connection. The PZT working frequency is related to the distance between the two electrode layers. The rectangular-prism-shaped PZT could reach $2 \mathrm{MHz}$ frequency provided the frequency constant of the PZT material is greater than 2000. Since the PZT prism had sharp edges and high absorption at $\lambda=$ $1550 \mathrm{~nm}$, it was polished to remove the sharp edges and coated with a thick layer of Teflon to form a low refractive index layer with a smooth surface and 'infinite' thickness compared to the microfiber diameter. Finally, a PZT rod with low refractive index layer was obtained, with a total diameter of $\sim 1.5 \mathrm{~mm}$.

The microfiber was wound around the coated PZT rod with three turns and then embedded in a low refractive index resin (UV373, manufactured by Luvantix, Korea). For additional protection, the two pigtails were totally embedded in a low refractive index polymer and the PZT modulator fixed on a glass slide. During the winding process, a minimal separation distance between the different turns was maintained to avoid unnecessary coupling between adjacent loops of the coil. This was achieved by winding the $\mathrm{OM}$ into a helix with a pitch of $100 \mu \mathrm{m}$. The schematic illustration and the photograph of the PZT OM phase modulator are separately shown in Fig. 2. It should be noted that the wound microfiber is very narrow and the coating layer is transparent, so neither is visible in Fig. 2(b).

In order to test its frequency response, the PZT OM phase modulator was inserted into one arm of a Mach-Zehnder interferometer, as shown in Fig. 3.

When a voltage potential is imposed between the electrodes of the PZT OM phase modulator, the cross section of the PZT rod deforms. This leads to a change in its circumference, resulting in stretching of the microfiber wrapped around it. The phase modulation along the microfiber is realized by varying the voltage $V$ imposed on the PZT electrodes. For small $V$, the phase modulator re-

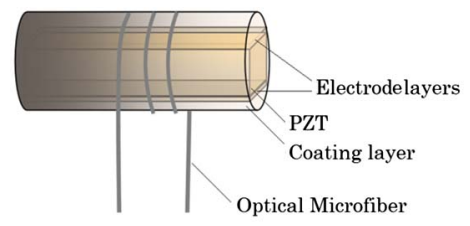

(a)

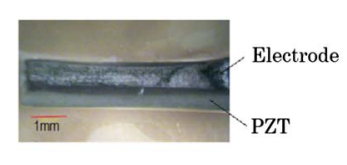

(b)
Fig. 2. (Color online) Schematic illustration (a) and photograph (b) of the PZT OM phase modulator.

sponse can be approximated as being linear and the induced phase along the OM can be expressed as

$$
\phi=K V,
$$

where $K$ is the voltage-phase transmission coefficient.

Figure 3 shows that a reference PZT modulator is used in the second arm of the Mach-Zehnder interferometer to produce a testing signal or a phase bias. Light from a laser source at $\lambda=1.55 \mu \mathrm{m}$ was injected into the interferometer, through an isolator and a polarization controller; the output of the interferometer was connected to a photodetector. The light intensity detected at the output of the interferometer can be expressed as

$$
I=A+B \cos \theta(t)
$$

where $\theta(t)$ is the time dependent phase difference between the interferometer arms. The constants $A$ and $B$ are proportional to the input optical power and can be obtained when $\theta(t)$ is swept over more than $2 \pi$. The interferometer visibility can be defined as

$$
v_{i}=B / A
$$

If a sinusoidal modulation with a frequency $\omega_{s}$ is imposed on the measured OM phase modulator in the interferometer, then Eq. (2) becomes

$$
I=A+B \cos \left[\phi_{s} \cos \left(\omega_{s} t+\varphi_{0}\right)+\phi_{0}(t)\right]
$$

where $\phi_{s}, \varphi_{0}$ are the amplitude and the initial phase of the measured phase signal and $\phi_{0}(t)$ represents environmental effects, which also include the initial phase difference between the two arms and the bias phase difference from the testing PZT modulator. When $\phi_{0}(t)$ is set to be equal to $\pi / 2$ by the testing PZT modulator, Eq. (4) can be simplified into

Microfiber PZT modulator

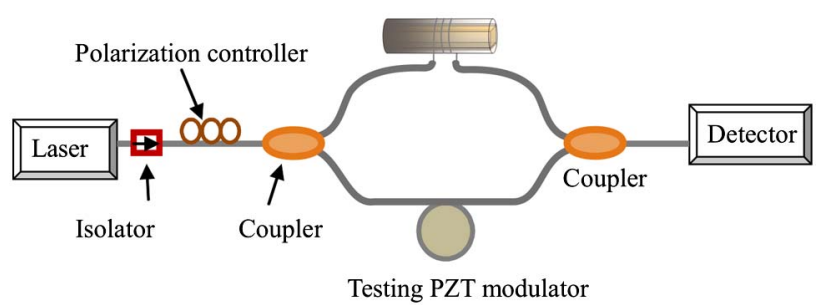

Fig. 3. (Color online) Schematic diagram of the measurement system. 


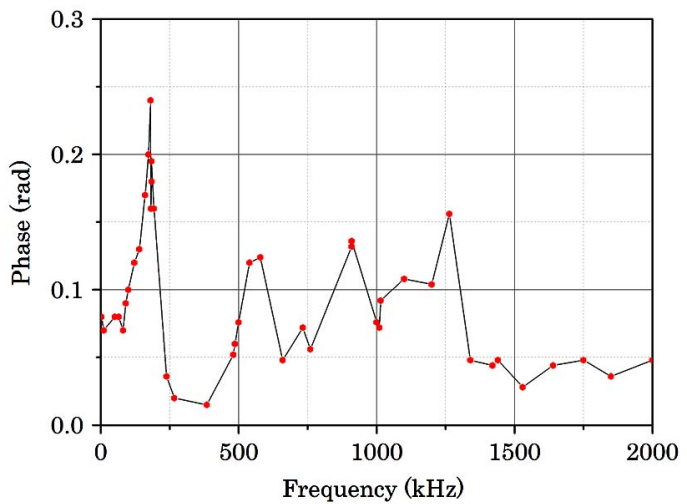

Fig. 4. (Color online) Measured frequency response of the PZT OM modulator.

$$
I=A-B \sin \left[\phi_{s} \cos \left(\omega_{s} t+\varphi_{0}\right)\right] .
$$

For sinusoidal modulation amplitude of less than $\pi / 10$, Eq. (5) can be simplified to

$$
I=A-B \phi_{s} \cos \left(\omega_{s} t+\varphi_{0}\right)
$$

Through a high-pass filter, Eq. (ㅁ) becomes

$$
I=B \phi_{s} \cos \left(\omega_{s} t+\varphi_{0}\right) .
$$

Then the amplitude $\phi_{s}$ of the phase signal with frequency $\omega_{s}$ can be measured by an oscilloscope, which can be obtained with

$$
\phi_{s}=I / B
$$

The frequency response of the PZT microfiber phase modulator as measured by detecting the phase change across a range of frequencies, as shown in Fig. $\underline{4}$. The amplitude of the signal imposed on the modulator was $9.3 \mathrm{~V}$.

The PZT microfiber phase modulator operated up to $2 \mathrm{MHz}$, with a natural resonating frequency of $\sim 180 \mathrm{kHz}$. The high-order resonating frequencies are approximately at three times $(\sim 540 \mathrm{kHz})$, five times $(\sim 920 \mathrm{kHz})$, and seven times $(\sim 1260 \mathrm{kHz})$ the natural frequency of the sample. The average phase response is about $0.07 \mathrm{rad}$ prior to the resonating frequency and $0.04 \mathrm{rad}$ near $2 \mathrm{MHz}$.

The length of OM used in the PZT modulator is $14 \mathrm{~mm}$ and the imposed amplitude signal is $9.3 \mathrm{~V}$; the responding efficiency of the modulator at the first resonating frequency, before the resonating frequency, and near $2 \mathrm{MHz}$ are $1.76 \mathrm{rad} / \mathrm{V} / \mathrm{m}, 0.53 \mathrm{rad} / \mathrm{V} / \mathrm{m}$, and $0.3 \mathrm{rad} / \mathrm{V} / \mathrm{m}$, respectively. Results show that the PZT microfiber phase modulator has a responsivity similar to conventional fiber-based PZT modulators, but with the additional benefits of having broader bandwidth and a smaller size.

The frequency was fixed at $186 \mathrm{kHz}$ to measure the phase change in response to varying the amplitude of the voltage signal imposed on the modulator. The experimental results are plotted in Fig. 5, clearly showing that the modulator has a good linear responsivity at the measured frequency.

The OM sample used to demonstrate the PZT phase modulator had high loss (almost $10 \mathrm{~dB}$ ). The nonuniform

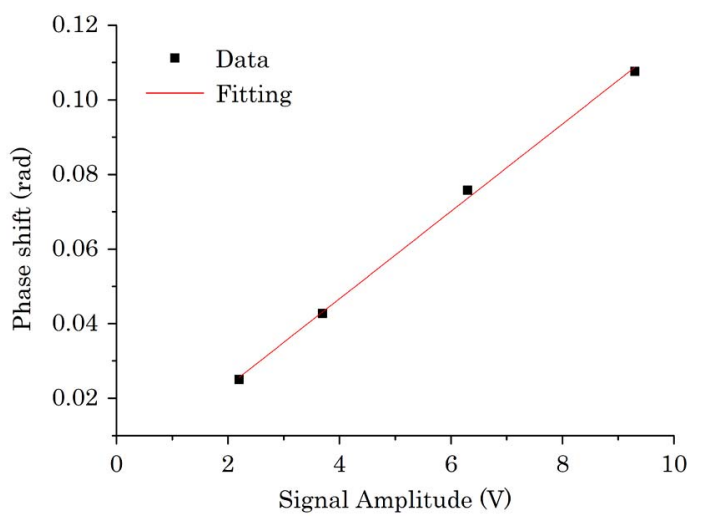

Fig. 5. (Color online) Measured (dots) and fitted (line) linear response of the $\mathrm{PZT}$ OM modulator at $186 \mathrm{kHz}$ frequency.

curing of the polymer, undesirable strain, and microbending along the microfiber are key reasons for the high loss of the sample. The focus of our work will be on improving the precision of the OM winding, the packaging technique, and the quantitative UV curing process, so as to minimize optical losses in the PZT OM modulator.

Furthermore, the OM phase modulator can also be improved with the development of longer OMs [12], which will increase the total responding efficiency of the OM modulator by allowing longer OM to be wound around the PZT rod. It is envisaged that the OM phase modulator can be very useful in applications of OM interferometers $[\underline{13}, \underline{14}]$.

In conclusion, OM technology enabled the demonstration of a PZT phase modulator exhibiting compact size, low-cost, and bandwidth near $2 \mathrm{MHz}$.

G. Brambilla gratefully acknowledges the Royal Society (London, U.K.) for his University Research Fellowship.

\section{References}

1. E. L. Wooten, K. M. Kissa, A. Yi-Yan, E. J. Murphy, D. A. Lafaw, P. F. Hallemeier, D. Maack, D. V. Attanasio, D. J. Fritz, G. J. McBrien, and D. E. Bossi, IEEE J. Quantum Electron. 6, 69 (2000).

2. S. A. Clarka, B. Cuishawa, E. J. C. Dawnayb, and I. E. Days, Proc. SPIE 3936, 16 (2000).

3. D. Jackson, R. Priest, A. Dandridge, and A. Tveten, Appl. Opt. 19, 2926 (1980).

4. G. Martini, Opt. Quantum Electron. 19, 179 (1987).

5. F. Trowbridge and R. Phillips, Opt. Lett. 6, 636 (1981).

6. N. H. Ky, H. G. Limberger, R. P. Salathe, and G. R. Fox, J. Lightwave Technol. 14, 23 (1996).

7. M. Imai, S. Satoh, T. Sakaguchi, K. Motoi, and A. Odajima, IEEE Photon. Technol. Lett. 6, 956 (1994).

8. D. M. Costantini, H. G. Limberger, R. P. Salathe, C. A. P. Muller, P. Muralt, and N. Setter, Appl. Phys. Lett. 78, 2193 (2001).

9. G. Brambilla, J. Opt. 12, 043001 (2010).

10. H. Yu, S. Wang, J. Fu, M. Qiu, Y. Li, F. Gu, and L. Tong, Appl. Opt. 48, 4365 (2009).

11. D. Marcuse, J. Opt. Soc. Am. 66, 216 (1976).

12. N. Vukovic, N. G. R. Broderick, M. Petrovich, and G. Brambilla, IEEE Photon. Technol. Lett. 20, 1264 (2008).

13. Y. Li and L. Tong, Opt. Lett. 33, 303 (2008).

14. G. Y. Chen, X. L. Zhang, G. Brambilla, and T. P. Newson, Opt. Lett. 36, 3669 (2011). 